\title{
Femtosecond laser fabrication of fiber based optofluidic platform for flow cytometry applications
}

\author{
Murat Serhatlioglu ${ }^{\mathrm{a}, \mathrm{b}}$, Caglar Elbuken ${ }^{\mathrm{a}, \mathrm{b}}$, Bulend Ortac $^{\mathrm{a}, \mathrm{b}}$, Mehmet E. Solmaz*c \\ ${ }^{a}$ UNAM - National Nanotechnology Research Center, Bilkent University, 06800, Ankara, Turkey; \\ ${ }^{\mathrm{b}}$ Institute of Materials Science and Nanotechnology, Bilkent University, 06800, Ankara, Turkey; \\ ${ }^{c}$ Department of Electrical and Electronics Engineering, Izmir Katip Celebi University, 35620, Izmir, \\ Turkey
}

\begin{abstract}
Miniaturized optofluidic platforms play an important role in bio-analysis, detection and diagnostic applications. The advantages of such miniaturized devices are extremely low sample requirement, low cost development and rapid analysis capabilities. Fused silica is advantageous for optofluidic systems due to properties such as being chemically inert, mechanically stable, and optically transparent to a wide spectrum of light. As a three dimensional manufacturing method, femtosecond laser scanning followed by chemical etching shows great potential to fabricate glass based optofluidic chips. In this study, we demonstrate fabrication of all-fiber based, optofluidic flow cytometer in fused silica glass by femtosecond laser machining. 3D particle focusing was achieved through a straightforward planar chip design with two separately fabricated fused silica glass slides thermally bonded together. Bioparticles in a fluid stream encounter with optical interrogation region specifically designed to allocate $405 \mathrm{~nm}$ single mode fiber laser source and two multi-mode collection fibers for forward scattering (FSC) and side scattering (SSC) signals detection. Detected signal data collected with oscilloscope and post processed with MATLAB script file. We were able to count number of events over $4000 \mathrm{events} / \mathrm{sec}$, and achieve size distribution for $5.95 \mu \mathrm{m}$ monodisperse polystyrene beads using FSC and SSC signals. Our platform shows promise for optical and fluidic miniaturization of flow cytometry systems.
\end{abstract}

Keywords: Flow cytometry, femtosecond laser, machining, hydrodynamic focusing, all-fiber, optofluidic, fused silica, microfluidics.

\section{INTRODUCTION}

Flow cytometers (FCs) firstly appeared in the literature around $1960 \mathrm{~s}^{1}$. There are many successful industrial products and research articles based on flow cytometers. It plays and important role in the diagnosis and monitoring of various diseases by inspecting, counting, and sorting particles and cells. Important physical and chemical parameters such as particle size and shape, granularity, DNA and protein content are extracted using the interaction of optical light beam while particles passing through flow medium. FCs are considered as an important clinical research tool for malignant epithelial cell immunophenotyping ${ }^{2}$ and CD4 T-lymphocyte counting in HIV detection ${ }^{3}$.

An FC consists four main parts: fluid delivery system, optic components, (laser diode, lenses, filters, photodiodes, fibers) electronic parts, (amplifier circuits, signal processing units, computer) and additionally, cell sorting system for specific applications. Fluid delivery system plays an important part since the particles in a FC system need to be aligned in 3D to the center of flow medium in order to avoid clogging and trans-off axial data acquisition. In a flow cytometer 3D focused particles are directed through a flow cell. During their passage from optical interrogation region, they scatter the incident excitation laser light to detectors which are specifically arranged for extracting different features.

*mehmete.solmaz@ikc.edu.tr; phone 90232 329-3535; fax 90232 329-3999; www.solmazresearchgroup.com

Optical Fibers and Sensors for Medical Diagnostics and Treatment Applications XVII, edited by Israel Gannot, Proc. of SPIE Vol. 10058, 100580I · @ 2017 SPIE · CCC code: 1605-7422/17/\$18 · doi: 10.1117/12.2252092 
The oppositely placed front detector collects forward scattered light (FSC), which is related to cell size and cell surface area. The angled detector/s collect side scatter light (SSC) which is related to cell granularity and complexity. Also fluorescent signal detection from fluorescently labeled cells occurs at side scattering using band pass filters for specific emission wavelengths. State of the art FC systems use bulk optics which have alignment issues, require large sample volumes, are expensive to buy and maintain, and requires qualified user for utilization and maintenance. In the last two decades, there has been great interest in microfluidic lab-on-a-chip systems towards fast bio-analysis and examination while using vastly smaller sample volume. Microfluidic based FCs (MFC) have attracted a great deal of interest in microfluidic system because they are advantageous for aspects of miniaturization, low-cost fabrication and production, small amount of sample requirement, high throughput in the measurement, and operating without a need for qualified operator. It also enables integration of optical elements into one single chip, which is a great opportunity for optical miniaturization.

Microfluidic devices are commonly fabricated with polymers, silicon and glass. Polymers are cost effective and easy to fabricate but suffers from chemical stability for long duration or multiple use applications ${ }^{4}$. Silicon and glass shows better chemical and mechanical stability. However, silicon is opaque to visible light it does not give desired performance for visualization and hence not preferred for optofluidic applications. On the other hand, glass surfaces are chemically inert, mechanically stable, and optically transparent to a wide spectrum of light. Glass-based microfluidic system fabrication with well-known cleanroom fabrication techniques are generally considered costly and time consuming compared to polymer based chip fabrication, Fortunately, femtosecond laser micromachining assisted hydrofluoric acid (HF) etching of transparent glass structures, opens the doors of cost and time reduced fabrication of complex structures in fused silica for microfluidic channels ${ }^{4-6}$, and monolithic integrated devices ${ }^{7-9}$.

Mao et al. implemented microfluidic drifting based 3D hydrodynamic focusing system and integrated optical fibers in a miniaturized PDMS based $\mathrm{MFC}^{10}$. Testa et al. showed self-aligned 3D hydrodynamic focusing in a fiber based MFC system ${ }^{11}$ which constitutes the basis for our 3D hydrodynamic focusing flow cytometry chip. Watts et al. showed and integrated lens system MFC architecture on in order to increase the detection efficiency at light interrogation zone ${ }^{12}$. Jang et al. showed low cost electroosmotically driven flow cytometry chip for dual fluorescent detection although it is not enough for high throughput applications as it serves as a unique conceptual result ${ }^{13}$. Paie et al. implemented femtosecond laser inscribed optical waveguides and femtosecond laser machined buried 3D complex 3D hydrodynamic focusing structure in fused silica which served as microfluidic cell counter device ${ }^{5}$. Recently Zhao et al. showed a PDMS disposable focusing lens and fibers integrated MFC chip for red blood cell and platelet counting application ${ }^{14}$. The common denominator for given and all other examples in the literature is achieving simple and low cost flow cytometry device.

Here we report, femtosecond laser machined, fiber-based fused silica microfluidic based flow cytometer platform supported with 3D hydrodynamic focusing using only one sheath and one sample fluid inlet. We used $5.95 \mu \mathrm{m}$ polystyrene beads in order to measure the performance of device. While hydrodynamically focused $5.95 \mu \mathrm{m}$ polystyrene beads passing through fiber interrogation line we excited with $5 \mathrm{~mW}$ optical power and $405 \mathrm{~nm}$ wavelength blue laser and we measured FSC signal with photodetector and SSC signal with PMT then calculated coefficient of variation (CV) values. Afterwards, we theoretically showed how focused particle location and particle size variation effects the calculated CV results.

\section{EXPERIMENTAL METHODS}

\subsection{Fabrication}

We have reported our femtosecond laser assisted carving (FLAC) fabrication method and detailed steps which provides faster scanning rate for mostly low repetition rate femtosecond laser machining requirements ${ }^{15}$. Also reported article gives great deal of comparison with traditional glass micromachining techniques in terms of machining time, precision, surface roughness, feature size, repeatability, multi-processing capability. Traditional laser radiation techniques require to radiate all 3D shape and takes more time, dependent to volume of design while FLAC only requires side-walls and bottom surface radiation of the design and carving it out with assisted HF etching. In summary, fabrication of miniaturized optofluidic FC microchip starts with CAD design and follows by femtosecond laser irradiation step through high precision automated XYZ stage on a custom made femtosecond laser machining bench. Radiated samples were then immersed into HF solution in order to develop structures. Afterwards, two patterned fused silica slides were bonded to each other with fusion bonding using no adhesive layer with thermal annealing of approximately 7 hours in a furnace at 
$650^{\circ} \mathrm{C}$. Then, tubing for inlets and outlet were bonded. Finally, the excitation and collection optical fibers were inserted through fiber slots that are designed to approximately be the same size as $125 \mathrm{um}$ diameter fibers. The fibers were then fixed with UV curable polymer. FLAC fabrication steps are illustrated in Fig. 1.

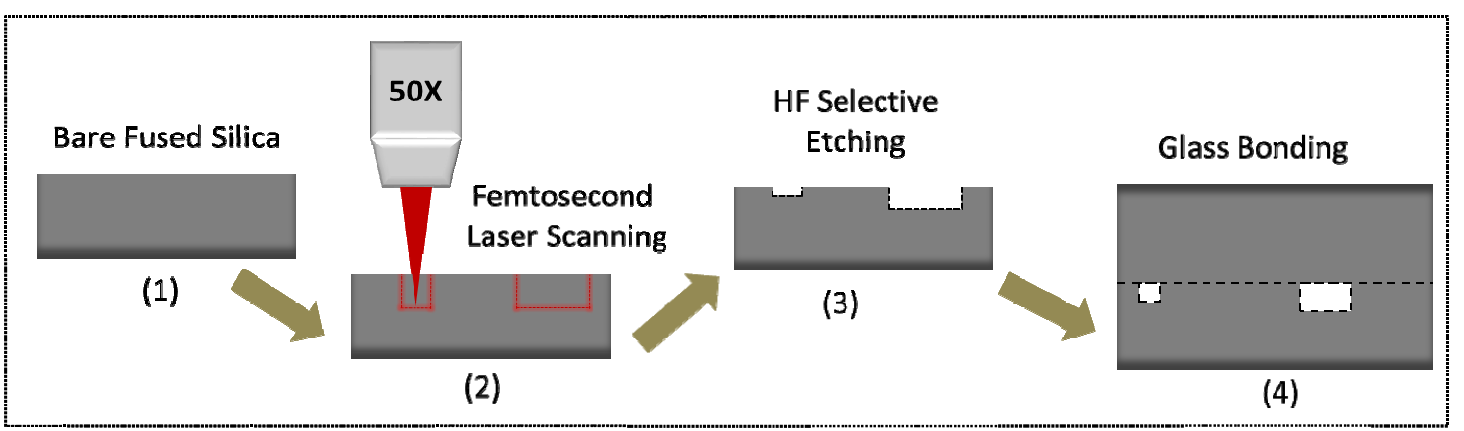

Figure 1. Illustrative drawing of the femtosecond laser machining of FC fabrication process.

Illustrative drawing of the patterned fused silica slides (Face 1 and Face 2) and the microscope images during FLAC process is given in Fig. 2. 3D hydrodynamic focusing arms and sample fluid surrounding micro-pool were divided into two parts and were carved on to both faces. Face 2 has the fiber grooves and sample fluid channel which has the same width with sheath flow arms but with half of the depth. Alignment holes at each corner were drilled using femtosecond with $3 \mathrm{~mm}$ diameter in order to achieve perfect alignment for bonding of both faces. Since micropool has a bigger size compared to channels, it was partitioned into smaller pieces for faster FLAC process. Face 2 images give the details of, center micropool as radiated (Fig. 2(b)), after 15 minutes of HF (Fig. 2(d)) and after 65 minutes of HF for center micropool (Fig. 2(e)), and the fiber grooves and main channel prior to the bonding step (Figs. 2(d)-2(g)).
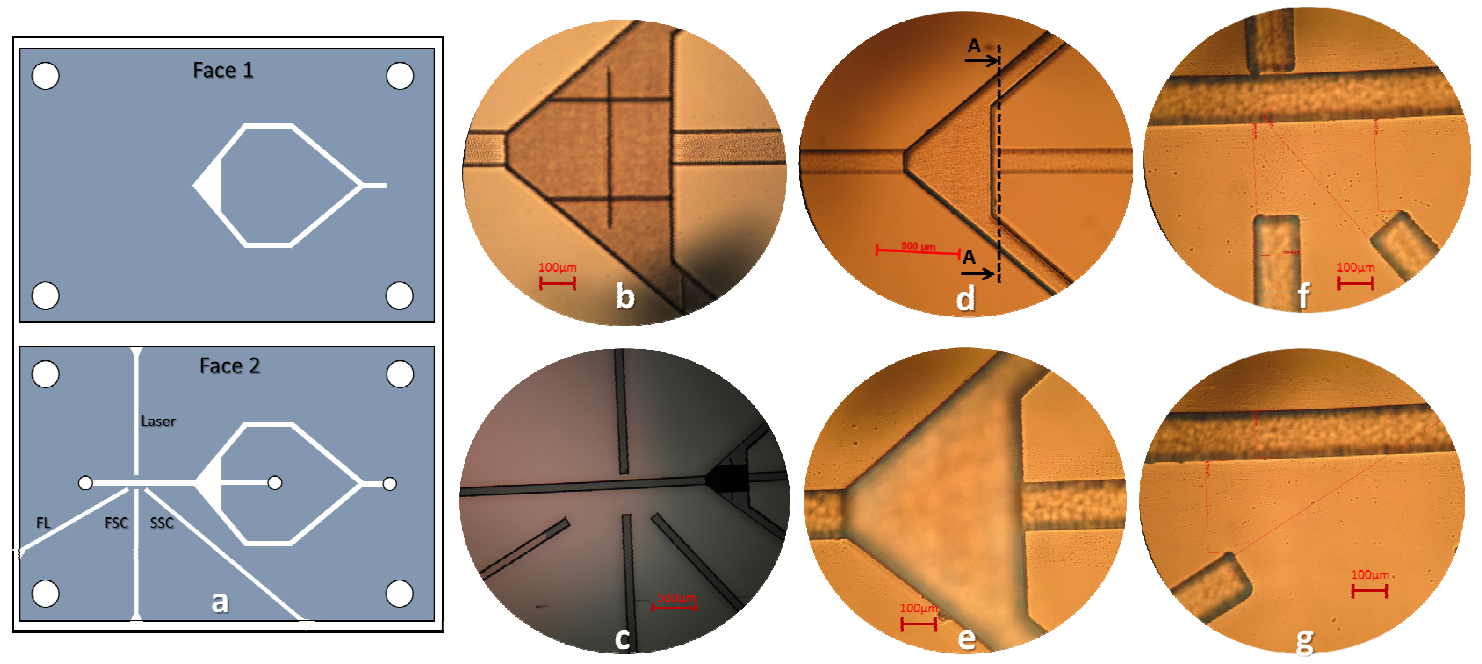

Figure 2. (a) Fabricated MFC design for both fused silica slides. Time-lapse microscope images for face 2 during HF etching, after (b) 0 min, (c) 15 minutes, (d)-(g) 65 minutes.

The size comparison of sheath arms and sample fluidic channels are given in Fig. 3, which is an illustrative drawing of A-A cross section in Fig. 2(d). Here the width " $w$ " and height " $h$ " is $\sim 140 \mu \mathrm{m}$ and the fiber grooves were designed to be the same size of sample channel. It is important to note that HF also etches bare fused silica at a slower rate ${ }^{16}$ and results in enlargement on desired dimensions. Hence the given dimensions were carefully planned and compensated in the channel design with regard to total HF etching time. 


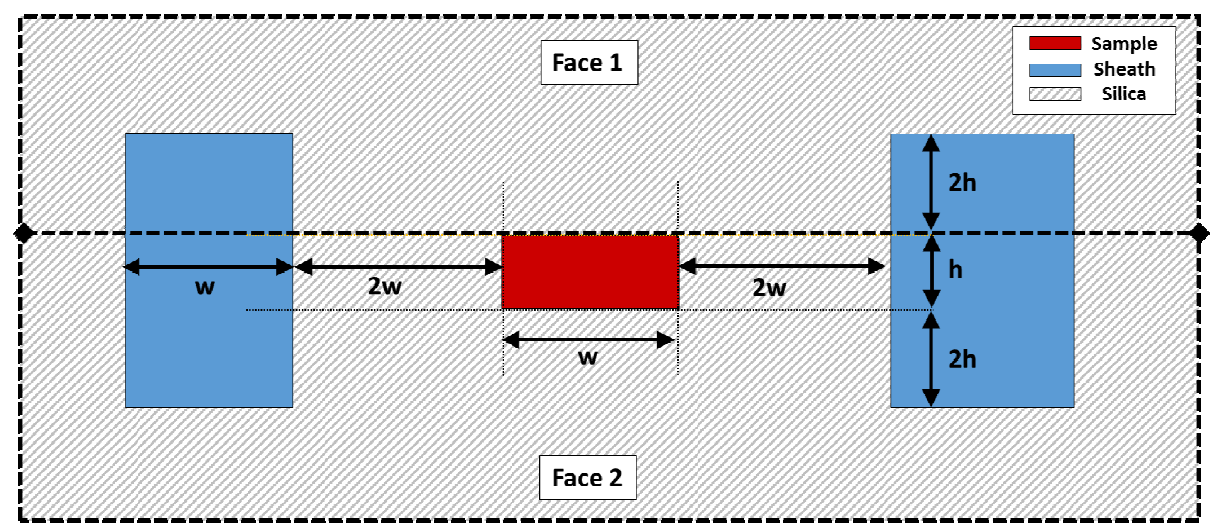

Figure 3. A-A cross section (in Fig. 2(d)) of the bonded FC chip. Sample channel 3D hydrodynamic focused in both vertical and horizontal directions.

\subsection{Optical methods}

An optical image was taken of the integrated, assembled, and ready to use $60 \times 20 \times 2 \mathrm{~mm}^{3}$ flow cytometry chip (Fig. 4(a)). As shown in illustrative drawing Fig. 4(b), the fiber-based FC chip includes four optical fibers: the excitation fiber and three detection fibers. The input fiber used was a single mode (SM) pigtailed blue fiber laser which is operated at $3 \mathrm{~mW}$, has a center wavelength of $405 \mathrm{~nm}$, numerical aperture (NA) of 0.12 , mode field diameter (MFD) of $3.6 \pm 0.5 \mu \mathrm{m}$, and is inserted perpendicularly to interrogation zone to guide the excitation light to the focused sample flow stream. The other three detection fibers are arranged at specific angles across the flow channel and are able to simultaneously collect detected FSC, SSC, and FL signals. FSC signal was detected with Thorlabs-FDS625 Graded Index pigtailed photodiode while we used Hamamatsu H7827-011 photomultiplier tube (PMT) for collecting the SSC signal. The center to center distance from the center point of focused sample stream is $\sim 10 \mu \mathrm{m}$ to laser excitation fiber, $\sim 300 \mu \mathrm{m}$ to FSC fiber which blocks the laser light when particles passing through, $\sim 500 \mu \mathrm{m}$ to SSC fiber, positioned at $140^{\circ}$ angle to excitation fiber and $\sim 800 \mu \mathrm{m}$ to FL fiber which was positioned at $120^{\circ}$ angle to the excitation fiber. We did not use any filter for FSC and SSC signal detection. Beside, we were not able to demonstrate the measurement for fluorescent detection, but fabricated the MFC chip capable of fluorescent signal detection with two different colors (535 and 710nm band pass filters) using fiber optical wavelength division multiplexing filters before PMTs as shown in Fig. 4(b).
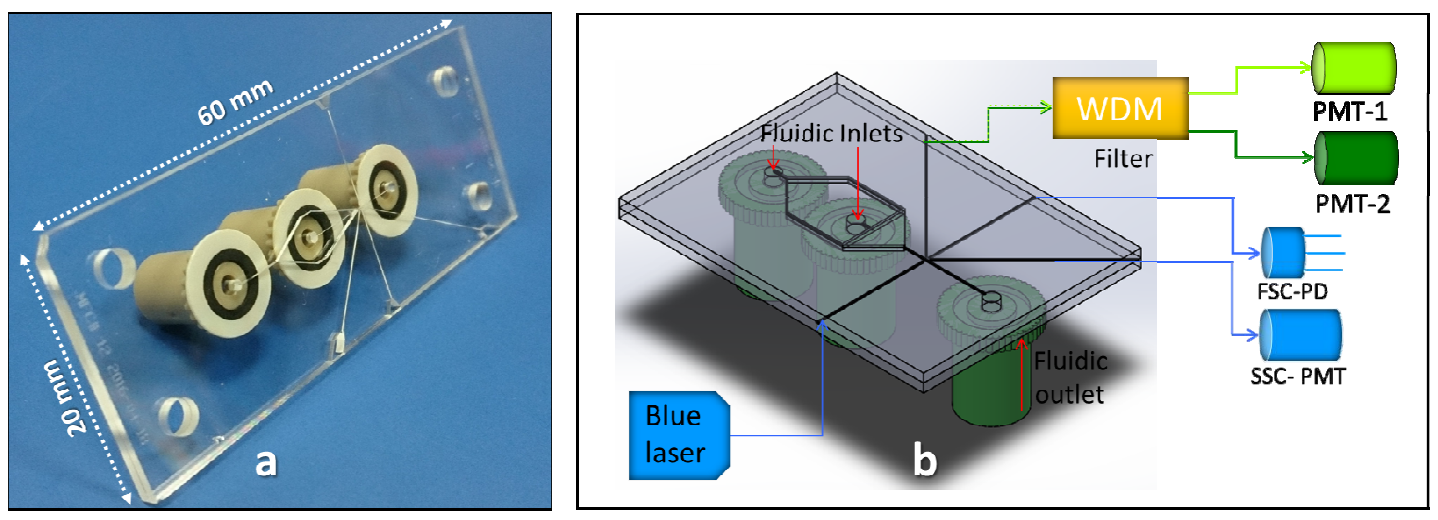

Figure 4. (a) Assembled and ready to use MFC chip; (b) The illustrative figure for input and output fiber assignments of the femtosecond laser fabricated MFC.

\subsection{Sample Preparation}

We used 1X phosphate buffered saline (PBS) as sheath fluid and $5.95 \mu \mathrm{m}$ polystyrene microbeads form Polysciences, Inc. at $0.12 \mu \mathrm{m}$ standard deviation (SD) used in the sample stream. The particles were diluted in a $1 \mathrm{X}$ PBS solution and blue colored food dye (filtered using $0.2 \mu \mathrm{m}$ pore syringe filter) for better visualization, to a concentration of $\sim 1000$ particles/ $\mu 1$ and sonicated for $15 \mathrm{~min}$ in order to avoid aggregation during experiments. 


\section{RESULTS AND DISCUSSION}

\subsection{Device performance tests}

The 3D hydrodynamic focusing performance of the FC chip was observed at different flow rates and results were shown in Fig. 5. PBS based sheath fluid was pumped through the sheath inlet at a pressure of $60 \mathrm{mbar}$ and hold stable, at the same time $5.95 \mu \mathrm{m}$ polystyrene bead mixture of solution was injected through the sample inlet at three different flow

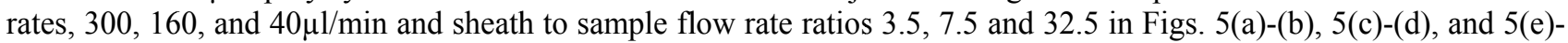
(f), respectively. Figures 5(b), 5(d) and 5(f) shows how sheath fluid squeezes the sample flow in micropool whereas Figs. 5(a), 5(c) and 5(e) shows the change in the diameter of focused stream in microchannel with varying sample fluid flow rate. The approximated cubic-diameter of the focused stream was measured as $\sim 35 \mu \mathrm{m}, \sim 17 \mu \mathrm{m}$ and $\sim 7 \mu \mathrm{m}$ in Figs. $5(\mathrm{a})$, 5 (c) and 5(e), respectively. Since our polystyrene particles are in $5.95 \mu \mathrm{m}$ size, $\sim 7 \mu \mathrm{m}$ focus diameter gives perfect resolution. Since sheath flow arms were fabricated symmetrically and merged in one inlet, 3D focusing always was kept at the center of channel and provided great stability to the performance of the device.
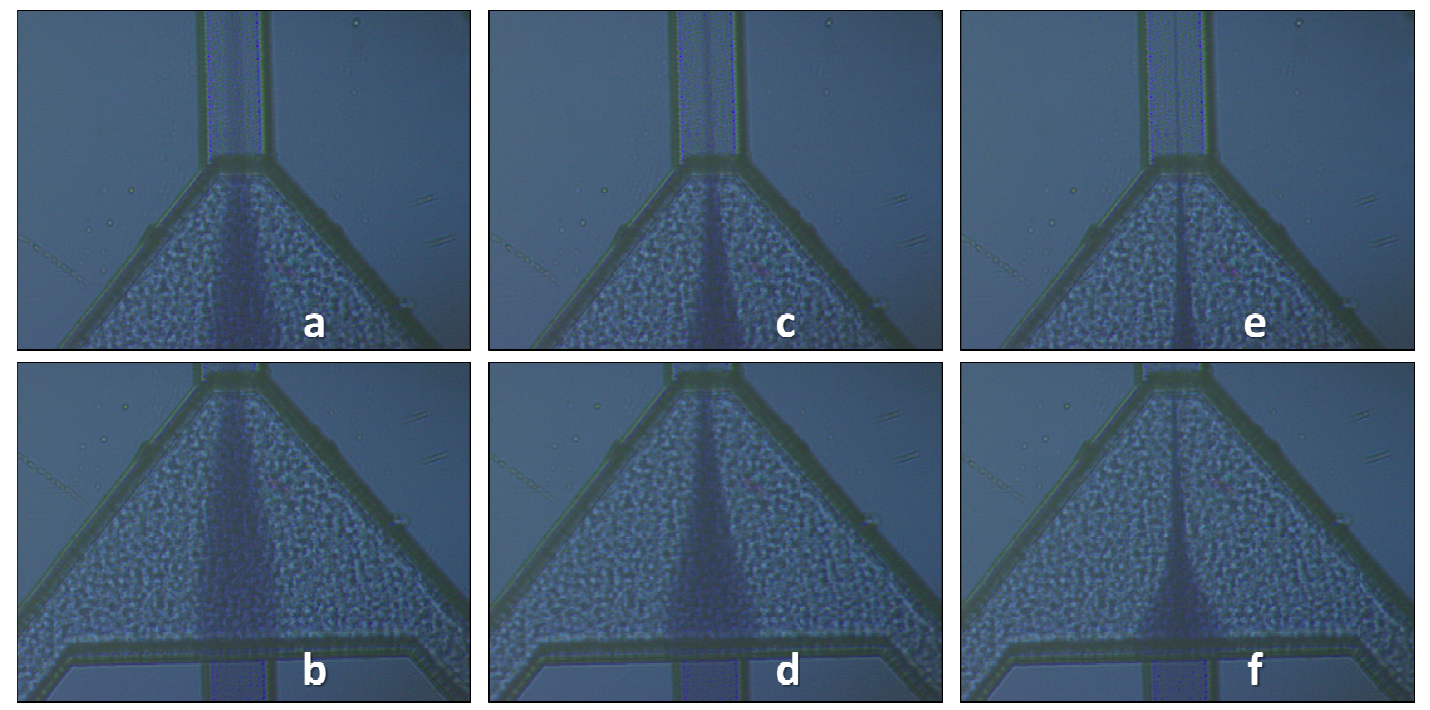

Figure 5. Self-aligned 3D hydrodynamic focusing with constant sheath flow rate but varying sample flow rate: (a), (c), (e) The change in the diameter of 3D focused sample stream; (b), (d), (f) 3D squeezing change in micropool. Sheath pressure is $\sim 60 \mathrm{mbar}$ (a)-(f). Sample flow rates; (a)-(b) $300 \mu \mathrm{l} / \mathrm{min}$; (c)-(d) $160 \mu 1 / \mathrm{min}$; (e)-(f) $40 \mu 1 / \mathrm{min}$.

FSC and SSC data were recorded simultaneously using a digital oscilloscope, for $200 \mathrm{~ms}$ at a sampling rate of $0.5 \mathrm{MHz}$ and then analyzed with a written script in MATLAB. Recorded signals for FSC and SSC are shown in Fig. 6; 200ms, $1 \mathrm{~ms}$ (zoomed view of $17^{\text {th }}-18^{\text {th }}$ milliseconds of recorded data) and $20 \mu \mathrm{s}$ (zoomed view of $17.39^{\text {th }}-17.41^{\text {st }}$ milliseconds of recorded data) in Figs 6(a), 6(b), and 6(c) respectively. Each detected peak is characteristic to size of the particles. Also the locations of FSC and SSC signals match with each other. As shown in $20 \mu$ s amplified graph, each peak has similar profile for FSC with pulse and SSC values. The time duration of each peak despite their height variation is $\sim 1 \mu \mathrm{s}$ and provides well-separated events at the limits of our system and would be enough for high throughput cytometry. Collected data was post-processed with MATLAB script and we resulted 846 events per $200 \mathrm{~ms}(\sim 4200 \mathrm{events} / \mathrm{sec})$. 

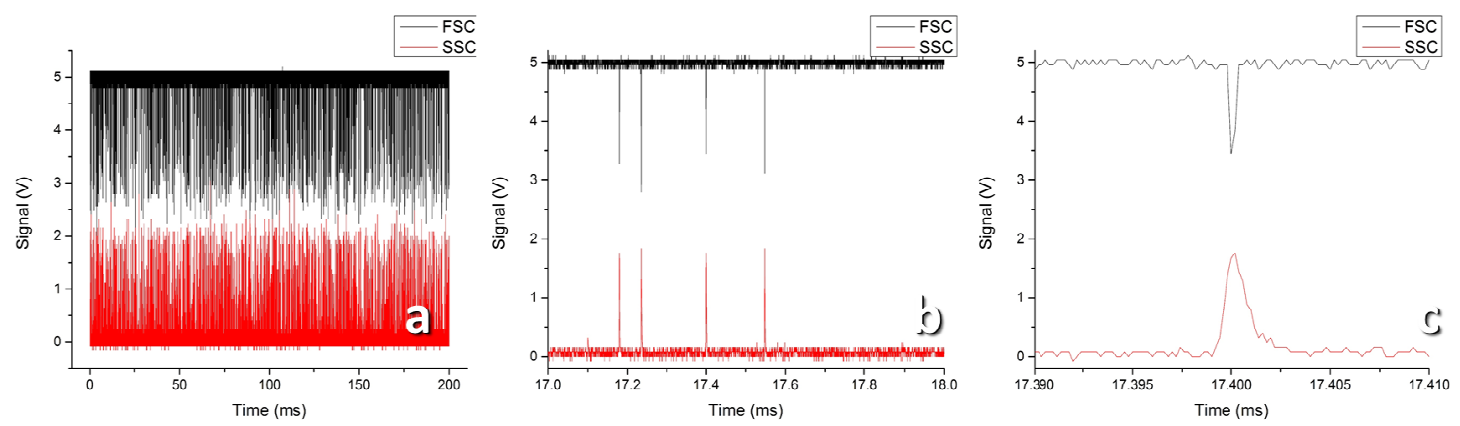

Figure 6. Recorded FSC and SSC signals prior to MATLAB processing step. (a) 200ms time duration; (b) 1ms time duration (zoomed data to $17^{\text {th }}$ to $18^{\text {th }}$ milliseconds recorded data); (c) $20 \mu \mathrm{ms}$ time duration (zoomed data in $17.39^{\text {th }}$ to $17.41^{\text {st }}$ milliseconds recorded data)

\subsection{Cytometry results and comparison with a commercial flow cytometry system}

We compared our test results with BD Accuri D6 flow cytometer commercially available for research facilities. We used 2D histogram plots for both FSC and SSC peak height values in Fig. 7 and calculated CV results with a comparison to commercial FC in Table 1. Measured voltage signal varies from 0 to $5 \mathrm{~V}$ for both FSC and SSC, however number of counted events amplify around $2 \mathrm{~V}$ for both signals. Even though the counted events amplify around 2V, both FSC and SSC have quite wide side wings which reach to $0 \mathrm{~V}$ in lower and $3 \mathrm{~V}$ and over in upper parts. This can be explained by the aggregated polystyrene bead doublets and residue particles in food dye. When we apply $80 \%$ gating to lower and upper region of side-wings and narrow down the calculation in between $1.4-2.5 \mathrm{~V}$, to exclude distortive false counts from the plot, we reached more accurate results and much lower $\mathrm{CV}$ values.
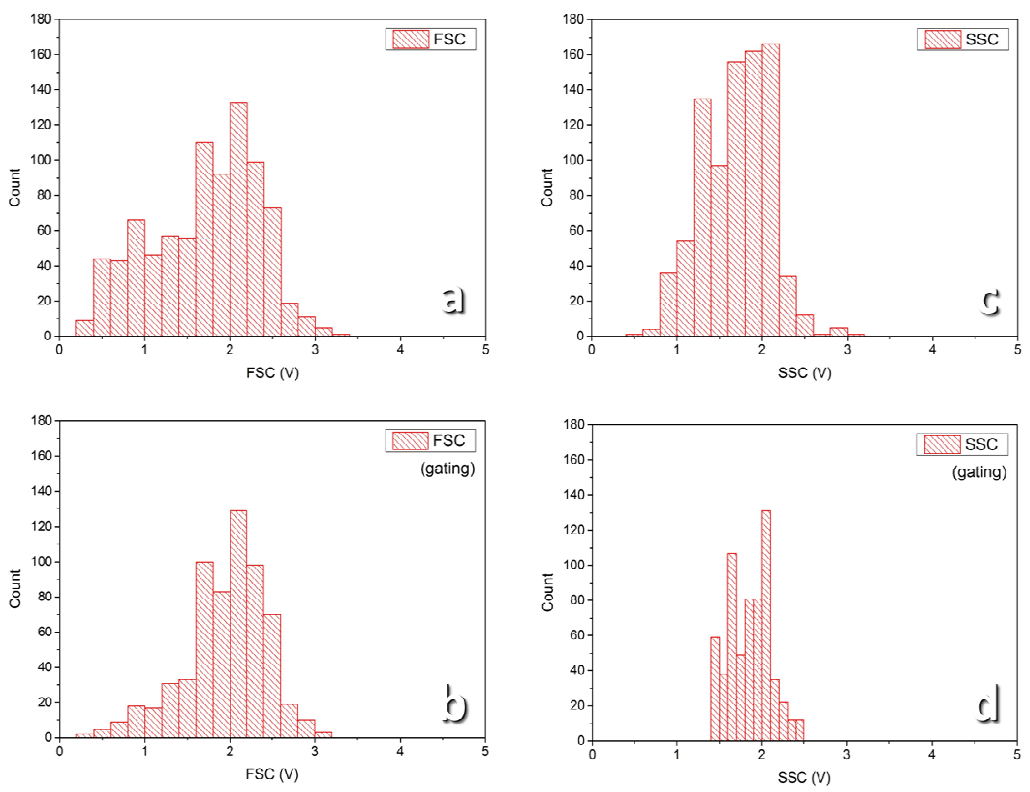

Figure 7. FSC and SSC signal peak histogram comparison for CV calculations of MATLAB post processed data. (a) Prior to gating for FSC signal; (b) after 80\% gating for FSC signal; (c) prior to gating for SSC signal; (d) after $80 \%$ gating for SSC signal.

Our FC chip has $37 \%$ CV for FSC signal and $22 \%$ CV for SSC signal before gating. When we apply $80 \%$ gating calculated CV values reduce to $24 \%$ and $13 \%$, respectively. On the other hand, the calculated results with the same sample in commercial BD Accuri FC, has 9\% CV for FSC signal and 37\% CV for SSC signal before gating. After we apply $88 \%$ gating calculated $\mathrm{CV}$ values reduce to $3.5 \%$ and $13 \%$, respectively. 
Experimental results show that the performance of commercial flow cytometer is better than the fabricated flow cytometry chip for FSC signal, and similar performance with gating for SSC values. A possible reason for worse performance could be very high sample flow rate and and low data sampling rate. Flow rate during experimental test of commercial flow cytometer was 20 times smaller than the flow rate for the fabricated MFC chip. Such high flow rate and low sampling rate can cause false data interpretation and signal variations for FSC values. Another reason could be due to the contribution of polystyrene bead size variation and imperfect alignment of particles in 3D hydrodynamic focusing. The beads used had a size variation of $5.95 \pm 0.12 \mu \mathrm{m}$. We performed a Monte-Carlo simulation on the effect of standard deviation $(0.12 \mu \mathrm{m})$ to the power variation on the detector (Fig. 8(b)). If a bead with $5.95 \mu \mathrm{m}$ in diameter passes perfectly at the center of $11 \mu \mathrm{m}$ wide optical beam (which is calculated using Gaussian beam propagation), the power variation is $0 \%$. However, assuming a Gaussian distribution for particle size, the light incident on the fiber also is a Gaussian distribution with a CV of $9.72 \%$. Even a small particle size variation can cause up to $10 \%$ change in signal height. Furthermore, we assumed that the particles might also be passing slightly off axis (Fig. 8(a)) with a standard deviation of $2 \mu \mathrm{m}$. Again, based on a Gaussian distribution of particle mislocation, the light on the detector gains a CV of $51.33 \%$ (Fig. 8(c)). According to the simulation results, particle off-axis passing and size distribution are major causes for obtaining FSC and SSC signals with a distribution.

Table 1. Comparison of CV values for fabricated MFC chip and commercial BD Accuri D6 flow cytometry instrument.

\begin{tabular}{|c|c|c|c|c|}
\hline Features & \multicolumn{2}{|c|}{ FC Chip } & \multicolumn{2}{|c|}{ BD Accuri D6 } \\
\hline $\begin{array}{l}\text { \# of events/sec } \\
\text { (before gating) }\end{array}$ & \multicolumn{2}{|c|}{$\sim 4200$ events $/ \mathrm{sec}$} & \multicolumn{2}{|c|}{$\sim 240$ events $/ \mathrm{sec}$} \\
\hline \# of total events during test & \multicolumn{2}{|c|}{$\sim 860$ events } & \multicolumn{2}{|c|}{192,790 events } \\
\hline Test duration & \multicolumn{2}{|c|}{200 milliseconds } & \multicolumn{2}{|c|}{13.5 minutes } \\
\hline Gating & Before Gating & $\begin{array}{c}\text { After Gating } \\
(\sim 80 \% \text { gating })\end{array}$ & Before Gating & $\begin{array}{l}\text { After Gating } \\
\text { ( } \sim 8 \% \% \text { gating) }\end{array}$ \\
\hline$\% \mathrm{CV}$ for FSC Signal & 37 & 24 & 9 & 3.5 \\
\hline$\% \mathrm{CV}$ for SSC Signal & 27 & 14 & 37 & 13 \\
\hline
\end{tabular}

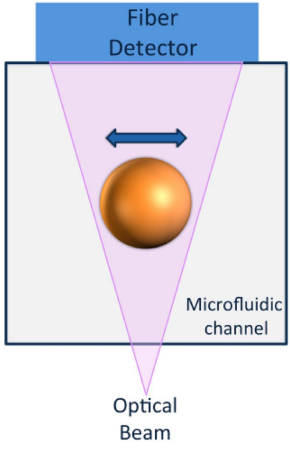

(a)

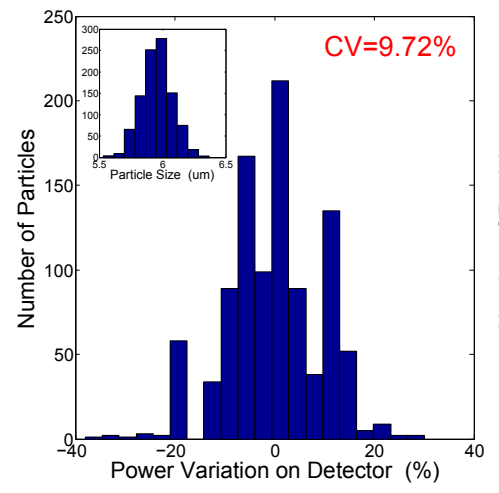

(b)

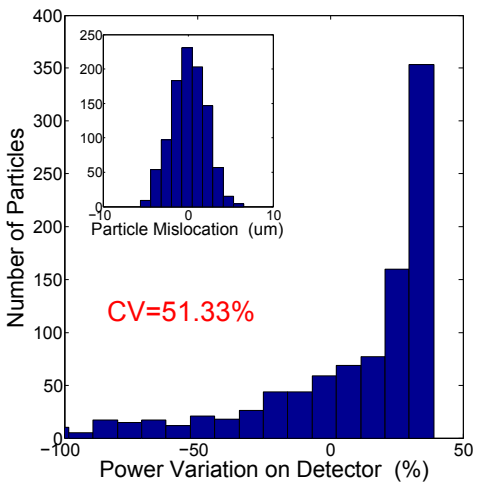

(c)

Figure 8. (a) Bead passing through the optical beam and momentarily blocking it. Moving the bead left-right causes the optical power on fiber detector to deviate; (b) The effect of particle size distribution on detector power variation; (c) The effect of particle mislocation on detector power variation.

\section{CONCLUSION}

In this study, we presented the design, fabrication and FSC-SSC characterization of fused silica fiber-based on-chip optical detection flow cytometry platform. A very narrow single stream of particles was achieved by self-aligned 3D hydrodynamic focusing with only one sheath and one sample inlet. Stream size variation at a fixed sheath flow rate and variable sample flow rate was achieved between $7-35 \mu \mathrm{m}$. We achieved over $\sim 4000$ events/sec throughput for detected events, at the limits of our system under present conditions. The CV values, flow rate, and number of counted events 
were compared to a commercial flow cytometer. We performed the test $\sim 20$ times faster than commercial flow cytometer at given conditions. Our platform gives relatively close results for SSC CV calculations while lacking the FSC CV calculations. We elaborated on the possible reasons of FSC CV difference in comparison. Applying further adjustments and changes to our fabricated chip could provide a higher throughput, low-cost microfluidic based flow cytometry platform for point-of-care use.

\section{ACKNOWLEDGEMENT}

This work is supported by The Scientific and Technological Research Council of Turkey (TUBITAK) under grant no. 113E321. Mehmet E. Solmaz acknowledges the EU COST action MP1205. Bulend Ortac acknowledges the TUBAGEBIP program. Authors also would like to acknowledge, Ph.D. candidate Nuray Gunduz for her tremendous support during BD Biosciences Accuri D6 flow cytometer measurements and great discussions during the data analysis.

\section{REFERENCES}

[1] Fulwyler, M. J., "Electronic Separation of Biological Cells by Volume," Science (80-. ). 150(3698), 910-911 (1965).

[2] Crow, M. J., Marinakos, S. M., Cook, J. M., Chilkoti, A.., Wax, A., "Plasmonic flow cytometry by immunolabeled nanorods," Cytom. Part A 79(1), 57-65 (2011).

[3] Cheng, X., Irimia, D., Dixon, M., Sekine, K., Demirci, U., Zamir, L., Tompkins, R. G., Rodriguez, W.., Toner, M., "A microfluidic device for practical label-free CD4(+) T cell counting of HIV-infected subjects.," Lab Chip 7(2), 170-178 (2007).

[4] Jagannadh, V. K., Mackenzie, M. D., Pal, P., Kar, A. K.., Gorthi, S. S., "Imaging flow cytometry with femtosecond laser-micromachined glass microfluidic channels," IEEE J. Sel. Top. Quantum Electron. 21(4), 1-6 (2015).

[5] Paiè, P., Bragheri, F., Vazquez, R. M.., Osellame, R., "Straightforward 3D hydrodynamic focusing in femtosecond laser fabricated microfluidic channels.," Lab Chip 14(11), 1826-1833 (2014).

[6] He, S., Chen, F., Yang, Q., Liu, K., Shan, C., Bian, H., Liu, H., Meng, X., Si, J., et al., "Facile fabrication of true three-dimensional microcoils inside fused silica by a femtosecond laser," J. Micromechanics Microengineering 22(10), 105017 (2012).

[7] Bellini, N., Vishnubhatla, K. C., Bragheri, F., Ferrara, L., Minzioni, P., Ramponi, R., Cristiani, I.., Osellame, R., "Femtosecond laser fabricated monolithic chip for optical trapping and stretching of single cells," Opt. Express 18(5), 4679-4688 (2010).

[8] Yang, T.., Bellouard, Y., "Monolithic transparent 3D dielectrophoretic micro-actuator fabricated by femtosecond laser," J. Micromechanics Microengineering 25(10), 105009, IOP Publishing (2015).

[9] Athanasiou, C. E.., Bellouard, Y., "A monolithic micro-tensile tester for investigating silicon dioxide polymorph micromechanics, fabricated and operated using a femtosecond laser," Micromachines 6(9), 1365-1386 (2015).

[10] Mao, X., Nawaz, A. A., Lin, S. C. S., Lapsley, M. I., Zhao, Y., McCoy, J. P., El-Deiry, W. S.., Huang, T. J., “An integrated, multiparametric flow cytometry chip using 'microfluidic drifting' based three-dimensional hydrodynamic focusing," Biomicrofluidics 6(2), 1-9 (2012).

[11] Testa, G., Persichetti, G.., Bernini, R., "Micro flow cytometer with self-aligned 3D hydrodynamic focusing.," Biomed. Opt. Express 6(1), 54-62 (2015).

[12] Watts, B. R., Zhang, Z., Xu, C.-Q., Cao, X.., Lin, M., "Integration of optical components on-chip for scattering and fluorescence detection in an optofluidic device," Biomed.Opt.Express 3(11), 2784-2793 (2012).

[13] Jiang, H., Weng, X.., Li, D., "Dual-wavelength fluorescent detection of particles on a novel microfluidic chip.," Lab Chip 13(5), 843-850 (2013).

[14] Zhao, Y., Li, Q., Hu, X.., Lo, Y., "Microfluidic cytometers with integrated on-chip optical systems for red blood cell and platelet counting," Biomicrofluidics 10(6), 64119 (2016).

[15] Solmaz, M. S. and B. O. and C. E. and N. B. and M. E., "CO 2 laser polishing of microfluidic channels fabricated by femtosecond laser assisted carving," J. Micromechanics Microengineering 26(11), 115011 (2016).

[16] Bellouard, Y., Said, A., Dugan, M.., Bado, P., "Fabrication of high-aspect ratio, micro-fluidic channels and tunnels using femtosecond laser pulses and chemical etching,” Opt. Express 12(10), 2120 (2004). 